\title{
Evaluation of University Campus Landscape Based on AHP: A Case Study of Southwest University of Science and Technology
}

\author{
Yi Liu \\ School of Civil Engineering and Architecture, Southwest University of Science and Technology, Mianyang, China \\ Email: 849839767@qq.com
}

How to cite this paper: Liu, Y. (2021) Evaluation of University Campus Landscape Based on AHP: A Case Study of Southwest University of Science and Technology. Open Access Library Journal, 8: e7784.

https://doi.org/10.4236/oalib.1107784

Received: July 24, 2021

Accepted: August 10, 2021

Published: August 13, 2021

Copyright $\odot 2021$ by author(s) and Open Access Library Inc.

This work is licensed under the Creative Commons Attribution International License (CC BY 4.0).

http://creativecommons.org/licenses/by/4.0/

\begin{abstract}
In order to quantitatively evaluate the qualitative problem of campus landscape comfort of Southwest University of Science and Technology, based on the principles of landscape design, the Analytic Hierarchy Process (AHP method) and comprehensive index method are used to establish a reasonable overall landscape evaluation model for the university campus. Firstly, professional teachers are invited to compare the importance of various factors of the landscape to determine the weight of each evaluation factor, and then conduct a field survey on various factors of the landscape of Southwest University of Science and Technology, and select representative sample plots for questionnaire survey and statistics. Finally, the comfort score evaluation and comprehensive evaluation grade analysis of the overall campus landscape are carried out. The results show that the campus landscape in the Southwest University of Science and Technology is generally better, and there is still much room for improvement. By summarizing and analyzing the existing problems of the campus landscape and putting forward suggestions for optimization, it aims to provide a certain reference for the future campus landscape construction.
\end{abstract}

\section{Subject Areas}

Architecture

\section{Keywords}

Campus Overall Landscape, Analytical Hierarchy Process (AHP), Landscape Evaluation

\section{Introduction}

University campus landscape is a very important part of colleges and universi- 
ties, an important carrier of cultural spirit inheritance, and a very important factor that affects the campus image and the lives of teachers and students [1]. The university campus environment plays an important auxiliary role in the dissemination of knowledge, and subtly promotes the campus academic atmosphere and humanistic atmosphere [2] [3]. Therefore, scientific research on the comprehensive evaluation of university campus landscape not only saves human and financial resources and builds an ecological campus, but also has important significance for the next stage of landscape improvement and transformation of the school, and it also has a certain reference value for other university landscape evaluations.

At present, the domestic application of analytic hierarchy process and comprehensive evaluation method to evaluate and analyze the campus landscape is mainly concentrated on the campus plant landscape [4]. There are relatively few studies on the overall campus landscape, and there are few related to the campus landscape design and comfort. The research conducted by Bai Yuansheng and others on the landscape evaluation of the plant community on the Mailu Campus of Jiangxi University of Finance and Economics emphasized the significance of plant spatial hierarchical structure, variety diversity and plant ornamental characteristics improvement or transformation [5]; Liu Yang and others on the winter campus plant landscape of Northeast Forestry University analyze the construction of campus landscape in specific seasons [6]; Lin Rui et al. evaluated the campus landscape of Yunnan Agricultural University from the aspects of ecological structure, aesthetic value, and psychological impact [7]. This paper uses the Analytic Hierarchy Process (AHP method) to try to quantify the impact of various landscape factors in the entire landscape system. This article takes Southwest University of Science and Technology as an example, and uses the hierarchical analysis method (AHP method) to establish a quantitative comprehensive evaluation system for college landscape comfort. Finally, the survey data is analyzed to obtain a quantitative evaluation of the campus landscape in the new campus of Southwest University of Science and Technology, and to provide an idea for the conclusion of the university landscape design. The problems to be solved in the article are as follows:

1) Use the analytic hierarchy process (AHP method) and the comprehensive index method to establish a reasonable overall landscape evaluation model of the university campus.

2) Investigate the different levels of satisfaction of campus teachers and students with the campus landscape of Southwest University of Science and Technology.

3) Through the analysis of the scoring results of the criterion layer and the evaluation factor layer, the shortcomings of the campus landscape design and suggestions for improvement are summarized.

\section{Method}

The research object of this paper is Southwest University of Science and Tech- 
nology. Southwest University of Science and Technology is located in Mianyang City, Sichuan Province. The campus is adjacent to the Second Ring Road of Mianyang City to the south, to the planned science, education and cultural area to the north, and to the science and innovation park around it. Various professional departments and gymnasiums are located on the periphery of the school, the teaching area is in the center of the school, the living area is in the south of the teaching area, and the sports field and gymnasium are on the east. The functional areas such as learning, living and sports are connected by greening, forming a coherent campus environment. The school campus occupies an area of 5463 acres, which is a hilly hinterland, and the overall shape is a trapezoid wide from the west to the east. On the whole, Southwest University of Science and Technology already has a more humane environment space and a more ecological landscape environment, but there are still problems with the overall campus landscape, and there is still much room for improvement in landscape construction.

This research combines relevant knowledge of campus landscape evaluation, analytic hierarchy process, campus landscape construction, etc., combs and integrates relevant literature, and consults related professional teachers, combined with the actual situation of the Southwest University of Science and Technology campus, uses analytic hierarchy process to establish a reasonable university campus. The landscape evaluation factor set, and the comprehensive index method is used to establish a hierarchical model of the overall landscape evaluation of the university campus. Using questionnaires to conduct statistical investigations on the various factors of the university campus landscape, and using the AHP method to perform hierarchical sorting, consistency testing and average weight analysis on the survey data to obtain the average weight of each factor, and perform comfort score, the comfort level of this campus is evaluated, and a quantitative evaluation of the overall landscape of the university campus is obtained. According to the evaluation results of the overall campus landscape, suggestions for the landscape renovation of the Southwest University of Science and Technology are proposed.

\section{Evaluation Indices and Weights}

The overall landscape evaluation system of the Southwest University of Science and Technology is proposed, and the hierarchical structure model of the overall landscape evaluation of the university campus is established. The survey data is sorted by hierarchy, consistency test and average weight analysis by AHP method and the average weight of each factor is obtained.

\subsection{Evaluation Indices}

On the basis of collecting a large amount of information and asking relevant professional teachers, graduate students, etc., appropriate index factors are selected. At the same time, with reference to landscape science, ecology and other 
disciplines, combined with the specific status quo of the site, the thinking progresses from abstract to concrete to abstract. On the basis of reference and analysis of the existing research and literature results, combined with the construction principle of the tomographic structure model, the overall landscape evaluation content is divided into 3 levels: objective level, criterion level and evaluation factor level. According to the overall landscape design principles, the criterion layer is further divided into 16 evaluation factors based on feature, aesthetics, ecology, and culture, forming the Southwest University of Science and Technology AHP campus landscape evaluation model (Figure 1).

\subsection{Questionnaire Survey}

Follow the principles of representativeness, objectivity, and randomness of sample selection, choose a variety of different types of landscape plots, combine the evaluation factors in the hierarchical structure model, design questionnaires, and evaluate the campus comfort with a score of 1 to 5 points, and the corresponding scores for the degree are: very bad (1 point), bad ( 2 points), normal (3 points), good (4 points), very good (5 points). At the same time, teachers of the gardening profession are invited to compare the evaluation factors in the hierarchical structure model, and calculate and determine the weight of each factor. According to the landscape comprehensive evaluation index method, the comprehensive score and evaluation grade of the sample plot are finally calculated.

The comprehensive evaluation method is used to obtain the comprehensive evaluation score. The formula is $B=\Sigma F_{i} \times X_{i}$, where $B$ represents the comprehensive evaluation index of a certain landscape, $X_{i}$ represents the weight value of a certain evaluation factor when considering various indicators, and $F_{i}$ represents a certain landscape in a certain landscape. The score value under the evaluation

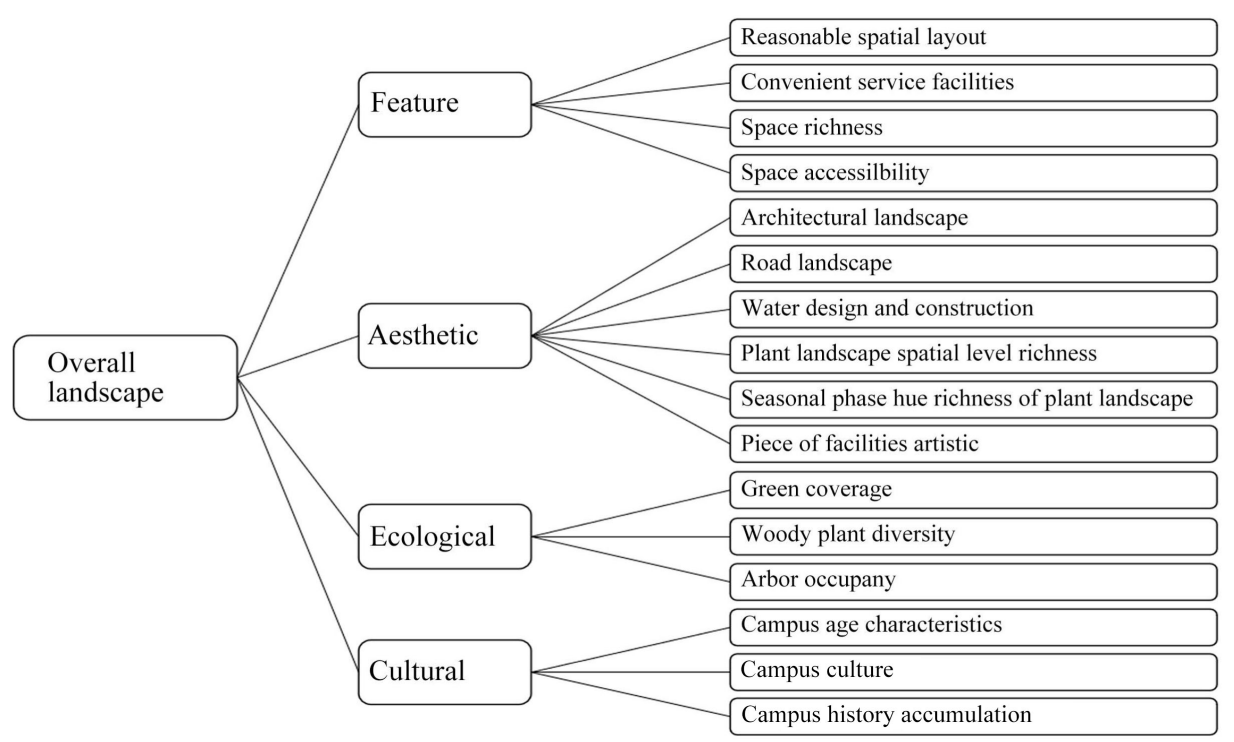

Figure 1. Overall landscape evaluation system of southwest university of science and technology. 
factor. The difference percentage method is used to classify [8], and the comprehensive comfort level of the landscape is divided into grades I, II, III, and IV (Table 1). The calculation formula is CWI $=\mathrm{S} / \mathrm{S} 0 \times 100 \%$, where CWI is the comprehensive evaluation index, $S$ is the landscape evaluation score value, and $\mathrm{S} 0$ is the ideal value (indicating that the highest level of each evaluation factor is multiplied by the weight and then added).

The investigation period is from November to December 2019. During the research, a total of 112 questionnaires were distributed, 112 of which were recovered, and 112 valid questionnaires were returned. The significance of the questionnaire survey is to directly obtain the information of the feelers, evaluate the comfort level, and provide effective feedback on the design quality and construction management.

\subsection{Calculating Weights of Evaluation Indices}

The analytic hierarchy process is a multi-objective decision-making analysis method that combines qualitative and quantitative analysis proposed by the American operations researcher Thomas L. Saaty in the 1970s [9] [10]. It is currently one of the most mature and widely used evaluation methods at home and abroad. It is suitable for problems that are difficult to fully quantitatively analyze. According to the weight calculation steps of the analytic hierarchy process: 1) Construct a paired judgment matrix. This paper uses the 1 - 9 scale method (Table 2) to construct the paired judgment matrix of the Southwest University of Science and Technology campus landscape comfort evaluation. First construct the judgment matrix $\mathrm{A} \sim \mathrm{B}$ of the criterion layer to the target layer, and then construct the judgment matrix $\mathrm{B} \sim \mathrm{C}$ of the evaluation factor layer and the criterion layer. In the process of research, the respondents based on the current situation of the campus landscape construction of Southwest University of Science and Technology, combined with the 1 - 9 scale method for the judgment matrix requirements, compared and analyzed the factors of each layer, and obtained the evaluation pair judgment matrix. 2) Hierarchical ranking and consistency test. Calculate the maximum characteristic root $\lambda \max$, consistency index CI, and average random consistency index RI of each comparison matrix, and the results meet the consistency test. Then, calculate the relative weight of a factor in a certain layer relative to a factor in the previous layer, and sort them one by one (Table 3 ) In this study, 14 experts from the valid questionnaire were selected to compare the weights of the evaluation factors at each level, and the SPSS analytic hierarchy process software was used to perform hierarchical ranking, weight calculation and consistency testing. Take the weight average of 14 respondents as the weight value of each factor of the target layer. In this study, the analytic hierarchy process was used to evaluate the landscape of the new campus of Southwest University of Science and Technology in both qualitative and quantitative aspects. The evaluation results are more scientific, reasonable and intuitive. 
Table 1. Classification of comprehensive landscape comfort level.

\begin{tabular}{ccccc}
\hline Percentage difference ratio (\%) & $80 \leq$ CWI $\leq 100$ & $60 \leq$ CWI $<80$ & $40 \leq$ CWI $<60$ & CWI $<40$ \\
\hline Landscape comfort level & I & II & III & IV \\
\hline
\end{tabular}

Table 2. Relative importance scales of AHP.

\begin{tabular}{c} 
Scaling \\
1 \\
3 \\
5 \\
7 \\
Comparison of two factors, one factor is not important relative to the other. \\
Comparison of two factors, one factor is slightly relative to the other. \\
Comparison of two factors, one factor is important relative to the other. \\
Median 2, $4,6,8 \quad$ Intermediate value of the above adjacent judgment. \\
\hline
\end{tabular}

Table 3. AHP campus landscape evaluation model.

\begin{tabular}{|c|c|c|}
\hline Objective layer & Criteria layer & Evaluation factor layer \\
\hline \multirow{16}{*}{$\begin{array}{l}\text { Overall landscape } \\
\text { of Southwest } \\
\text { University of } \\
\text { Science and } \\
\text { Technology }\end{array}$} & & Reasonable space layout C11[0.1385] \\
\hline & Feature & Convenient service facility C12[0.1004] \\
\hline & $\mathrm{B} 1[0.4082]$ & Richness of space place C13[0.1271] \\
\hline & & Accessibility of space location accessibility C14[0.0422] \\
\hline & \multirow{6}{*}{$\begin{array}{l}\text { Aesthetic } \\
\text { B2[0.1989] }\end{array}$} & Architectural landscape C21[0.0317] \\
\hline & & Road landscape C22[0.0334] \\
\hline & & Water design and construction C23[0.0326] \\
\hline & & Richness of plant landscape spatial level C24[0.0369] \\
\hline & & Seasonal phase hue richness of plant landscape C25[0.0329] \\
\hline & & Artistic of sketch facilities C26[0.0314] \\
\hline & \multirow{3}{*}{$\begin{array}{l}\text { Ecological } \\
\mathrm{B} 3[0.2339]\end{array}$} & Green coverage C31[0.1053] \\
\hline & & Woody plant diversity C32[0.0665] \\
\hline & & Arbor occupancy C33[0.0621] \\
\hline & \multirow{3}{*}{$\begin{array}{c}\text { Cultural } \\
\text { B4[0.1590] }\end{array}$} & Campus age features C41[0.0461] \\
\hline & & Campus culture atmosphere C42[0.0492] \\
\hline & & Campus history accumulation C43[0.0637] \\
\hline
\end{tabular}

\section{Result}

\subsection{Analysis of Weight Calculation Results}

Weight refers to the degree of importance of the evaluation factor layer relative to the target layer. It is different from the general proportion. It reflects not only the percentage of a certain factor, but also emphasizes the relative importance of the factor, and tends to contribute or importance. In the AHP campus landscape evaluation model of Southwest University of Science and Technology established 
in this study, the highest weight in the criterion layer is functionality, followed by ecology and aesthetics, and the lowest is cultural (Table 3). It shows that in the design and construction of the campus landscape of Southwest University of Science and Technology, more attention should be paid to the importance of functionality, the construction of ecological benefits and the aesthetics of the landscape. The top three weights in the evaluation factor layer are the rationality of spatial layout, the abundance of spatial locations, and the coverage of greenery, indicating of that campus landscape construction should not only pay attention to reasonable layout, of but also consider the richness of the spatial and location landscape and the coverage of greenery. It is also an aspect that needs to be paid attention to in the process of constructing the university campus landscape.

\subsection{Evaluation Factor Layer Analysis}

A statistical analysis was made on the evaluation results of the Southwest University of Science and Technology campus landscape survey questionnaire (Figure 2), and the scores of all evaluation items were comprehensively compared. The evaluation factor layer (C31, C32, C33) under the criterion layer B2 all scored higher, and the highest score is the green coverage rate, indicating that the landscape ecology of the Southwest University of Science and Technology is recognized by teachers and students. After consulting the data, it is known that the campus is now There are more than 300,000 trees, more than 2 million shrubs, and 3500 bamboos. The green area rate is $45 \%$, the green coverage rate is $57.97 \%$, the per capita green area is about 36 square meters, the greening rate reaches $98 \%$, the campus woody plants, and the number of trees is large The green coverage rate of the campus is gratifying. During the field investigation, it was found that many landscapes on the campus were designed with green plants

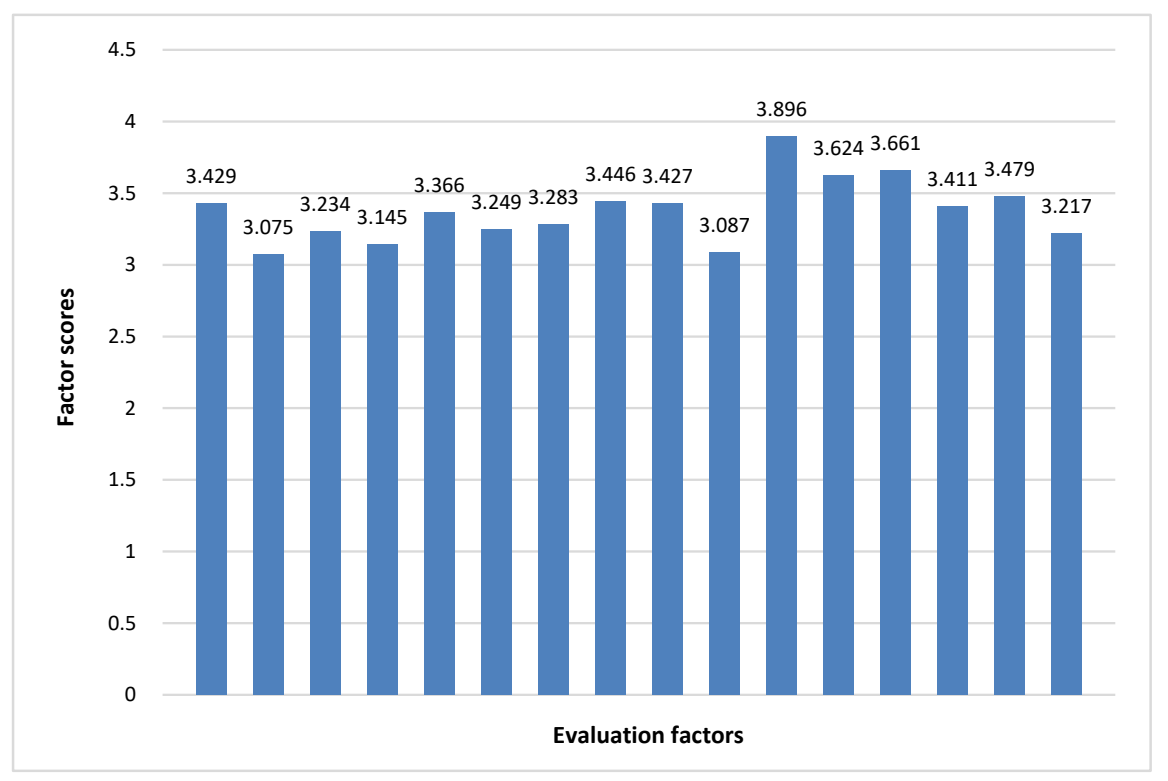

Figure 2. Evaluation result after single factor use. 
as the main body. Among them, the Central Lake-Sihai Lake area was very popular among teachers and students.

The lowest score is the convenience of service facilities (C12), indicating that students are very dissatisfied with the convenience of service facilities, especially the lack of public transportation facilities on campus in the new district, and the lack of service facilities such as large supermarkets in the new district. The lack of convenience of facilities has caused the inconvenience of students' life and study. In addition, the artistry (C26) of the sketch facilities under the criterion level B2 is also low. Although many architectural sketches have been built on the campus of Southwest University of Science and Technology, most of the sketches were constructed without public opinion, resulting in insufficient aesthetics of the sketches. At the same time, the facilities of the sketches in the old area of Southwest University of Science and Technology are too old, and their maintenance status has also led to the loss of aesthetics.

\subsection{Comprehensive Evaluation Result Analysis}

In the selected sample plots, a questionnaire was issued on the spot, allowing users to score 1 to 5 points for the comfort level of each landscape evaluation factor. Collect and sort out the comfort scores of each evaluation factor in 112 questionnaires, take the average value, and then calculate the comprehensive evaluation score of the plot according to the formula according to the average weight of each factor, and use the comprehensive evaluation index percentage for the score. The comfort grading method is used for grading.

$\mathrm{B} 1=3.429 \times 0.1385+3.075 \times 0.1004+3.234 \times 0.1271+3.145 \times 0.0422=$ 1.3274069;

$$
\begin{aligned}
\mathrm{B} 2 & =3.366 \times 0.0317+3.249 \times 0.0334+3.283 \times 0.0326+3.446 \times 0.0369+ \\
3.427 & \times 0.0329+3.087 \times 0.0314=0.6590821 ; \\
\mathrm{B} 3 & =3.896 \times 0.1053+3.624 \times 0.0665+3.661 \times 0.0621=0.8785929 ; \\
\mathrm{B} 4 & =3.441 \times 0.0461+3.479 \times 0.0492+3.217 \times 0.0637=0.5347198 ; \\
\mathrm{CWI} & =(1.3274069+0.6590821+0.8785929+0.5347198) / 5 \approx 68.00 \% .
\end{aligned}
$$

After calculation, the comprehensive evaluation index of Southwest University of Science and Technology is $68.00 \%$, and the evaluation level of landscape comfort is II. It shows that the Southwest University of Science and Technology has a good evaluation of the comfort of the landscape after use. The comprehensive evaluation results of the landscape factors of each criterion level: Feature (B1) > Ecological (B3) > Aesthetic (B2) > Cultural (B4).

\subsection{Criteria Layer Analysis}

Feature has the highest score among the criteria-level factors. From the single-factor evaluation of functionality and the statistical results of plot scores, it can be seen that the highest score is the reasonable spatial layout, followed by the richness of the space and the accessibility of the space, and the lowest evaluation is convenient service facilities. It shows that the campus landscape of Southwest University of Science and Technology is generally functional. The campus occu- 
pies a large area and is widely distributed, with a reasonable spatial layout. However, although there are many landscape creations, the layout of service facilities is ignored, and the practicability is not high. Moreover, the land is wide, many sites are not fully developed and utilized, and the service facilities in the park are few and scattered, and facilities and services cannot be provided in time. In addition, do a good job of site safety risk assessment to avoid safety problems.

From the single factor evaluation of aesthetics and the statistical results of the plot scores, it can be seen that the scores of the evaluation factors are between 3 and 4 points. The highest score is the richness of the plant landscape space, and the higher scores are the architectural landscape and the plant landscape season. For the richness of hue, the lowest scores are road landscape, water design and construction, and the artistry of sketches and facilities. The campus landscape of Southwest University of Science and Technology is better overall. In terms of plant landscapes, most plants grow vigorously and vigorously, with reasonable layout and configuration, complementing each other with other landscapes, and there are also rich and diverse green plant varieties. The community has distinct layers and rich seasonal changes. The group with lower comfort scores after use is composed of phoenix trees. Building roads and landscapes can meet the needs of daily life learning, but the problem of campus roads is relatively large, and there is often congestion in school and students, and students often seek low-grade roads. Therefore, when planning for the road landscape, more consideration should be given to the daily carrying capacity of the main road for students and teachers, and then the shaping of the landscape should be considered.

According to the evaluation results after ecological use, the students are relatively satisfied with the ecological characteristics of the campus landscape, and the average scores of all factors are above 3.5 points. The highest score is the green coverage, the higher score is the amount of arbor, and the lowest score is the diversity of grasses. In Southwest University of Science and Technology campus garden plants, arbor accounts for a larger portion, reaching $41.3 \%$; this reflects the planting principle of tall arbor as the key tree species in the garden landscape planting design, and the quality of the landscape created by the arbor is relatively high. Herbs and shrubs are next, accounting for $30.7 \%$ and $25.3 \%$ respectively; vines are the least, accounting for only $2.7 \%$. In vertical landscape design, vines are the best material, but vines are the least used, highlighting the problem of less vertical greening on the campus of Southwest University of Science and Technology, and the need to increase the intensity of three-dimensional greening.

It can be seen from the evaluation results after cultural use that the highest score is the campus cultural atmosphere, the higher score is the characteristics of the campus era, and the lowest score is the campus history accumulation. The construction of the campus in the new district has only a short period of time. The design and construction of the campus focuses on spatial functional research and reflecting contemporary characteristics, and the historical culture has not 
penetrated much. In the process of campus planning and development, buildings with the characteristics of the times and cultural symbols are retained in the old district.

\section{Conclusion}

This study combines two survey methods, field visits and questionnaires, and uses analytic hierarchy and comprehensive index evaluation methods to evaluate the comfort of the campus landscape of Southwest University of Science and Technology. The results show that ecology and functionality are critical to creating a good campus landscape. The overall landscape comfort of the university campus is well evaluated. Starting from the principles of landscape design, this research conducts campus evaluation and analysis from four aspects: functionality, aesthetics, ecology, and culture. It is recommended that the overall landscape planning and design of the university campus should be considered comprehensively, based on the principles of landscape design, and following the concept of "people-oriented". We should not only pay attention to the beautiful landscape construction, but forget the functionality of the landscape layout, and make the functions of the landscape facilities meet the needs of teachers and students need.

\section{Conflicts of Interest}

The author declares no conflicts of interest.

\section{References}

[1] Forman, R.T.T. and Godron, M. (1986) Landscape Ecology. John Wiley \& Sons, New York.

[2] Dober, R.P. (1992) Campus Design. John Wiley and Sons, New York, 3-50.

[3] Kaplan, R. (1985) The Analysis of Perception via Preference: A Strategy for Studding How the Environment Is Experienced. Landscape Planning, 12, 161-176. https://doi.org/10.1016/0304-3924(85)90058-9

[4] Silva, P. (2020) Not So Much about Informality: Emergent Challenges for Urban Planning and Design Education. Sustainability, 12, Article No. 8450. https://doi.org/10.3390/su12208450

[5] He, X.Y., Song, L., Xu, W.D. and Xie, D. (2006) Application of AHP on Developing an Evaluation System for Tree Species of UF. Liaoning Forestry Science and Technology, Vol. 3, 1-15. (In Chinese)

[6] Bascetin, A. (2004) An Application of the Analytic Hierarchy Process in Equipment Selection at Orhaneli Open Pitcoal Mine. Transactions of the Institutions of Mining and Metallurgy-(AusIMM), Section A-Mining Technology, 113, 192-199. https://doi.org/10.1179/037178404225004968

[7] Zhang, H., Wu, J. and Sun, C.Z. (2008) Evaluation on Wetland Ecosystem Service in Liaoning Province. Resources Science, 30, 267-273.

[8] Brabyn, L. and Mark, D.M. (2011) Using View Sheds, GIS, and Landscape Classification to Tag Landscape Photograph. Applied Geography, 31, 1115-1122.

https://doi.org/10.1016/j.apgeog.2011.03.003 
[9] Saaty, T.L. (1994) Fundamentals of Decision Making and Priority Theory with the Analytic Hierarchy Process. RWS Publication, Pittsburgh.

[10] Saaty, T.L. and Vargas, L.G. (1994) Decision Making in Economic, Political, Social, and Technological Environments with the Analytic Hierarchy Process. RWS Publications, Pittsburg. 